\title{
Rheological and biological properties of a hydrogel support for cells intended for intervertebral disc repair
}

Karin Benz ${ }^{1}$, Claudia Stippich ${ }^{1}$, Claudia Osswald ${ }^{2}$, Christoph Gaissmaier ${ }^{2}$, Nicolas Lembert ${ }^{3}$, Andreas Badke ${ }^{4}$, Eric Steck, Wilhelm K Aicher ${ }^{6}$ and Juergen A Mollenhauer ${ }^{1,2,7^{*}}$

\begin{abstract}
Background: Cell-based approaches towards restoration of prolapsed or degenerated intervertebral discs are hampered by a lack of measures for safe administration and placement of cell suspensions within a treated disc. In order to overcome these risks, a serum albumin-based hydrogel has been developed that polymerizes after injection and anchors the administered cell suspension within the tissue.

Methods: A hydrogel composed of chemically activated albumin crosslinked by polyethylene glycol spacers was produced. The visco-elastic gel properties were determined by rheological measurement. Human intervertebral disc cells were cultured in vitro and in vivo in the hydrogel and their phenotype was tested by reverse-transcriptase polymerase chain reaction. Matrix production and deposition was monitored by immuno-histology and by biochemical analysis of collagen and glycosaminoglycan deposition. Species specific in situ hybridization was performed to discriminate between cells of human and murine origin in xenotransplants.

Results: The reproducibility of the gel formation process could be demonstrated. The visco-elastic properties were not influenced by storage of gel components. In vitro and in vivo (subcutaneous implants in mice) evidence is presented for cellular differentiation and matrix deposition within the hydrogel for human intervertebral disc cells even for donor cells that have been expanded in primary monolayer culture, stored in liquid nitrogen and reactivated in secondary monolayer culture. Upon injection into the animals, gels formed spheres that lasted for the duration of the experiments (14 days). The expression of cartilage- and disc-specific mRNAs was maintained in hydrogels in vitro and in vivo, demonstrating the maintenance of a stable specific cellular phenotype, compared to monolayer cells. Significantly higher levels of hyaluronan synthase isozymes-2 and -3 mRNA suggest cell functionalities towards those needed for the support of the regeneration of the intervertebral disc. Moreover, mouse implanted hydrogels accumulated 5 times more glycosaminoglycans and 50 times more collagen than the in vitro cultured gels, the latter instead releasing equivalent quantities of glycosaminoglycans and collagen into the culture medium. Matrix deposition could be specified by immunohistology for collagen types I and II, and aggrecan and was found only in areas where predominantly cells of human origin were detected by species specific in situ hybridization.
\end{abstract}

Conclusions: The data demonstrate that the hydrogels form stable implants capable to contain a specifically functional cell population within a physiological environment.

\footnotetext{
* Correspondence: juergen.mollenhauer@nmi.de

${ }^{1} \mathrm{NMI}$ Natural and Medical Sciences Institute at the University of Tuebingen,

Reutlingen, Germany

Full list of author information is available at the end of the article
} 


\section{Background}

Biological regeneration of degenerated or traumatically damaged intervertebral discs is a desirable goal, and in the meantime an experimental focus of regenerative medicine. In recent time, the re-injection into the damaged disc of autologous disc cells harvested from prolapses has been even performed in clinical trials $[1,2]$. While data from such trials are emerging and suggest at least partial clinical success, the procedure is technically not satisfying yet. The injection of a liquid suspension of cells into the disc tissue is not safe enough without any measures to warrant the cells to remain within the disc and not be ejected during initial loading of the disc upon patient motion. Beyond this safety aspect, the introduction of fresh cells may not deliver success without simultaneously restoring the nutritional requirements and the moderation or prevention of inflammatory insults.

This present situation led several research groups to consider hydrogels as a means to anchor cells in situ, with the specific advantage that hydrogels might improve the nutritional situation of a disc with respect to its water content and the additional mechanical benefits of rebuilding disc height and resistance to compression. Two main strategies are imaged in literature: the surgical implantation of a preformed implant (derived from cellfree applications of hydrogels), and the injection of in situ polymerizing formulations. The present situation is reviewed in $[3,4]$. The structural basis for cell-supplemented hydrogels span across a remarkably long list of molecular elements, including mammalian components such as gelatin, chondroitin sulfate, hyaluronan, collagen, plant polymers such as alginate, and synthetic polymers such as polyethylene glycol, poly glycolic acid, and polylactic acid or synthetic peptides. The crosslinking is achieved by redox reactions (thiols), condensations (polyacrylates), or complex formation (alginate, peptides). Some of those reactions are designed to exclude cellular components from untoward inclusion into the reaction cascade, thus avoiding toxic interactions; others are balanced to minimize reactions that include cell surface components, without being strictly selective. Cell survival has been reported to be good to excellent, with most of the reports initially relying on in vitro observations. From those, a limited number has already been applied in animal experiments, either in ectopic or intradiscal administrations, with so far none reported to generate local or systemic adverse reactions.

Within this broader context, we also developed a hydrogel to be injected as a fluid and to polymerize in situ. The hydrogel is based on maleinimide-activated human serum albumin and cross-links chemically by dithiol-polyethylene glycol after mixing in a dual chamber syringe system equipped with an interrupted-helical mixing chamber. The hydrogel is enhanced with hyaluronan (HA) and adjusted to a visco-elastic stage that allows the gel to creep rather than build up significant local pressure, thus intended to anchor cells within the defect compartment but not to rebuild disc volume. The hydrogel displays anti-angiogenic properties in cell culture and in chorioallantois membrane tests [5]. Those properties are considered to be key elements for disc regeneration since recently published data underscore the negative effects of capillary ingrowth into inflamed disc tissues [6-9]. The high molecular weight crosslinked HA (Visiol ${ }^{\circledR}$, TBS Chemedica, Munich) itself is passively interwoven with the albumin polymer since it does not participate in the polymerization reaction. HA is included in the recipe because of its well-described chondro-protective, anti-inflammatory and biomechanical benefits (for review, see [10]). Numerous other groups are therefore currently also considering to apply HA in tissue engineering of cartilaginous implants, alone or in combination with other materials, and in various stages of technical development [11-23].

Here we present first experimental evidence for the hydrogel to be a suitable carrier for anchoring of disc cells in an ectopic animal model. The hydrogel stabilizes a chondrogenic phenotype of the cells, and facilitates deposition of a cartilagenous extracellular matrix.

\section{Methods}

Animals

Subcutaneous implantation of the human preparation was performed in a double-homozygous severe combined immunodeficient (SCID) hairless outbred model (Crl:SHO-Prkdc ${ }^{\text {scid }} \mathrm{Hr}^{\mathrm{hr}}$ mice; Charles River Laboratories; Sulzberg, Germany). The animal experiment was approved by the Committee on Animal Care of the State of Baden-Wuerttemberg (Germany).

\section{Hydrogel preparation}

Activated human maleolyl-albumin, hyaluronan and cell culture medium without supplements and without serum were used as basis for the albumin gel. Cross-linking was achieved through a specific thio-polyethylene glycol ( $\mathrm{SH}-$ PEG, Rapp Polymere GmbH; Tuebingen, Germany) spacer via Michael reaction of a maleolyl-group to a $\mathrm{SH}$-group, as described before [24] (pending patents number: PCT/ EP2008/005643, DPMA 102008008 071.3).

For the production of maleolyl-albumin, $250 \mathrm{mg}$ of human serum albumin (Sigma-Aldrich; Steinheim, Germany) were dissolved in $5 \mathrm{ml} 1 \mathrm{M}$ sodium borate (Sigma-Aldrich). Afterwards $75 \mu \mathrm{l} 260 \mathrm{mM}$ maleolyl- $\beta$ alanine in PBS/sodium borate $(1: 1)$ were added and incubated for $90 \mathrm{~min}$ at room temperature. $106 \mathrm{mg}$ 3-maleimidopropionic acid N-hydroxysuccinimide ester (Obiter Research; Urbana, USA) were dissolved in $950 \mu \mathrm{l}$ dimethylformamide. $500 \mu \mathrm{l}$ of this solution were 
added to the albumin solution and incubated for another $60 \mathrm{~min}$. at room temperature. Afterwards $500 \mu \mathrm{l} 3 \mathrm{M}$ sodium acetate (Sigma-Aldrich) were added and dialysed against phosphate buffered saline (PBS) for $1 \mathrm{~h}$ on ice three times. The dialysate was concentrated to $3.5 \mathrm{ml}$ by ultrafiltration (YM-3 membrane, Millipore; Billerica, USA) and stored at $-80^{\circ} \mathrm{C}$.

To prepare $2 \mathrm{ml}$ of hydrogel solution, $140 \mu \mathrm{l}$ maleolylalbumin $(43 \mathrm{mM}), 1,060 \mu \mathrm{l}$ cell culture medium and $400 \mu \mathrm{l}$ high molecular weight hyaluronan $(20 \mathrm{mg} / \mathrm{ml}$, Visiol; TRB Chemedica AG, Munich, Germany) were mixed and incubated for $5 \mathrm{~min}$. at room temperature. The remaining $400 \mu \mathrm{l}$ were reserved for the cell suspension.

The crosslinker solution comprised of $500 \mu \mathrm{l}$ SH-PEG $(10,000 \mathrm{~g} / \mathrm{mol}, 15 \mathrm{mM} \mathrm{SH}$-groups) in $0.1 \mathrm{mM} \mathrm{HCl}$, to achieve a 1:1 ratio of maleolyl-groups to $\mathrm{SH}$-groups when being mixed with the hydrogel/disc cell solution. The gel mixtures were submitted to a DIN/ISO 10993-4, -5, 10, -11 biocompatibility testing (including gels prepared from human albumin and of the test species mouse, rabbit, rat, and guinea pig) by BIOSERV Analytik \& Medizinprodukte $\mathrm{GmbH}$ (Rostock, Germany) and passed successfully.

\section{Rheological measurement of the visco-elastic properties of the hydrogel and effect of storage of gel components on the gelation process}

All gel experiments were performed using a dual chamber syringe system, with a $2-\mathrm{ml}$ chamber filled with the human disc cells suspended in culture medium plus high molecular weight hyaluronan and the polymerization agent maleimide-activated albumin, the other chamber filled with the chemical crosslinker SH-PEG, as described before [5,24].

To examine the visco-elastic properties of the hydrogels and the effect of storage of gel components on the gelation process rheological measurements were performed with a Kinexus Pro rheometer (Malvern Instruments $\mathrm{GmbH}$; Herrenberg, Germany). Per analysis day the instrument performance was checked before the measurement procedure using calibration oil as a standard (PRA Coating Technology; Hampton, UK). The two gel components were mixed and injected onto the bottom plate of the rheometer using a dual-chamber applicator syringe as described in [24] and in the introduction. After automatically adjusting the upper plate $(1 \mathrm{~Pa}$, plane configuration, diameter $20 \mathrm{~mm}$, spacing $0.6 \mathrm{~mm}$ ) a ring envelope was mounted around the piston to prevent water evaporation during the experiment. The measurements started approximately $30 \mathrm{sec}$. after gel mixture. The experimental conditions for detection of gel formation were: temperature $37^{\circ} \mathrm{C}$, shear strain $0.05 \%$, shear stress $1 \mathrm{~Pa}$, oscillation frequency $1 \mathrm{~Hz}$. The gelation process was monitored for a period of $20 \mathrm{~min}$. The electronic system continuously detected and stored the storage modulus ( $\mathrm{G}^{\prime}$ in $\mathrm{Pa}$ ) and the loss modulus (G" in Pa) with a frequency of $1 \mathrm{~Hz}$. The pressure of the upper plate on the gel was set to $1 \mathrm{~Pa}$ and was automatically controlled during the process of gelation.

\section{Human tissue harvest}

For the SCID mouse experiment human intervertebral disc (IVD) tissues were harvested from six patients during spinal fusion surgery. The donors were between 18 and 61 years old, two of them were female and four were male. For the in vitro experiment, the IVD tissues were harvested from 2 patients undergoing nucleotomie after disc herniation (male, 37 years; female, 32 years) and 2 patients undergoing spinal fusion surgery (male, 46 years; female, 46 years).

Due to the nature of the surgical procedures, a clear distinction between annulus and nucleus pulposus tissue could not always be made. Therefore, we aimed to collect unspecified IVD cells rather than distinct populations. All human tissues were obtained from the BG Trauma Center in Tuebingen (Germany). The studies were approved by the local ethics committee and informed consent was obtained from all individuals participating in the study. The sterile tissue was placed into culture medium and instantly transported to the tissue culture facility.

\section{Cell culture}

\section{Human IVD cell isolation and culture}

Tissue samples were washed twice in PBS (BioWhittaker; Verviers, Belgium) and then minced by scalpels. Extracellular matrix was enzymatically degraded overnight by incubation in DMEM high glucose medium (Invitrogen; Karlsruhe, Germany) containing $0.375 \mathrm{mg} / \mathrm{ml}$ collagenase B (Roche; Mannheim, Germany), $0.05 \mathrm{mg} / \mathrm{ml}$ hyaluronidase (Serva; Heidelberg, Germany) and 5\% human serum at $37^{\circ} \mathrm{C}$. Isolated disc cells were filtered through a $100-$ $\mu \mathrm{m}$ cell strainer to remove undigested tissue fragments and extracellular matrix debris. After centrifugation, the cells were resuspended in DMEM/Ham's -F12 cell culture medium supplemented with $5 \%$ human serum (Centre for Clinical Transfusion Medicine, University of Tuebingen, Germany), $150 \mu \mathrm{M}$ ascorbic acid-2-phosphate, $100 \mathrm{U} / \mathrm{ml}$ penicillin, $100 \mu \mathrm{g} / \mathrm{ml}$ streptomycin and plated in $75 \mathrm{~cm}^{2}$ cell culture flasks at an initial density of $0.5 \times 10^{6}$ cells in a humidified atmosphere (95\% air, 5\% CO2). The cells were harvested at $80-90 \%$ confluence by trypsin-EDTA (BioWhittaker) treatment, washed, centrifuged, the cell pellet resuspended in serum-free medium, and the cell number determined (P0 culture). For the in vitro experiment, the cells were use directly for subsequent passage 1 (P1) cultivation either in monolayer or embedded in the hydrogel. For the in vivo experiment the P0 cells were stored above liquid nitrogen until further use. 


\section{In vitro experiment}

To compare the behavior of the disc cells in the hydrogels and in monolayer culture, $0.5 \times 10^{6}$ cells/500 $\mu \mathrm{l}$ hydrogel were cultured in one well of a 24-well plate. In parallel, $0.17 \times 10^{6}$ cells were seeded in $25 \mathrm{~cm}^{2}$ cell culture flasks. Cultures were harvested at day 4, 7, and 14 and used for gene expression analysis.

\section{In vivo experiment}

The frozen cells from all six primary cultures were simultaneously re-activated prior to the experiment by seeding them once more into $75 \mathrm{~cm}^{2}$ cell culture flasks at $2 \times 10^{6}$ cells/flask and expanding into confluence in the presence of 10\% human serum and 1:1000 ITS liquid media supplement (insulin, transferrin, sodium selenite). They were harvested simultaneously by trypsinization (P1 cells). The harvested human cells were suspended in the implantation culture medium (consisting of a proprietary mixture of good manufacturing practice (GMP) grade phenol red free culture medium, supplemented with $5 \%$ human serum), then mixed with the hydrogel solution $\left(5 \times 10^{6} / \mathrm{ml}\right.$ gel solution) and transferred into the 2-mlcompartment of a dual chamber syringe. The second 0.5-ml-compartment was filled with SHPEG solution. The syringe was maintained at $4^{\circ} \mathrm{C}$ until use.

\section{Surgical implantation into and recovery from mice}

Using the dual-chamber syringe, $1.25 \times 10^{6}$ IVD disc cells resuspended in $250 \mu \mathrm{l}$ hydrogel were injected subcutaneously in the back of double-homozygous SCID hairless outbred model (Crl:SHO-Prkdc ${ }^{\text {scid }} \mathrm{Hr}^{\text {hr }}$ mice; Charles River Laboratories; Sulzberg, Germany). Polymerization of the hydrogel was achieved in situ. Two weeks later, the hydrogels were explanted, lysed for gene expression analysis or embedded in Tissue Tek, snap-frozen in liquid nitrogen and stored. As a control, hydrogels containing IVD cells from the same patients were cultured in vitro (250 $\mu$ l in a 24-well) for two weeks ( $n=6$ patients).

\section{Histology}

The frozen tissue samples were sliced by a cryotome to prepare $50 \mu \mathrm{m}$ sections, and stained with haematoxylin-eosin (HE). The sections were analyzed by immunohistology with antibodies against collagen type I (Anti-human collagen type I 63170, lot 1467 K, MP Biomedicals; LLC, Solon, Ohio, USA), collagen type II (clone II-II6B3; $1 \mu \mathrm{g} / \mathrm{ml}$; [25], and aggrecan (SM1353, Lot 040308, Acris Antibodies GmbH; Herford, Germany). The collagen type II antibody was obtained from the Developmental Studies Hybridoma Bank maintained by the Department of Pharmacology and Molecular Sciences, Johns Hopkins University School of Medicine, Baltimore, MD 21205, and the Department of Biological Sciences, University of Iowa, Iowa City, IA 52242, under contract NO1-HD-2-3144. Secondary antibodies were Cy3-conjugated IgG antibodies from Dianova (Hamburg, Germany). Control sections were prepared without primary antibody. The stained sections were analyzed by normal light or fluorescence microscopy (Axiophot, Zeiss; Jena, Germany). Photography was performed with an electronic camera (Axiocam, Zeiss).

\section{In situ hybridization}

For the discrimination between cells of murine and human origin species specific in situ hybridization was done. Digoxigenin (DIG)-labeled mouse specific SINE/B1 and SINE/B2 and human specific Alu probes were generated as described previously [26]. Sections were washed three times in PBS containing $0.1 \%$ Tween at room temperature and then treated with $50 \mu \mathrm{g} / \mathrm{ml}$ proteinase $\mathrm{K}$ (Fermentas, Germany) in PBS containing $0.1 \%$ Tween for $10 \mathrm{~min}$ at $37^{\circ} \mathrm{C}$. After washing in PBS, sections were treated with $0.25 \%$ acetic acid containing $0.1 \mathrm{M}$ triethanolamine $(\mathrm{pH}$ 8.0) for $10 \mathrm{~min}$. and then pre-hybridized for $1 \mathrm{~h}$ at $42^{\circ} \mathrm{C}$ in hybridization buffer containing $4 \times$ saline-sodium citrate (SSC), $50 \%$ deionized formamide, $1 \times$ Denhardts solution, $5 \%$ dextrane sulfate and $100 \mu \mathrm{g} / \mathrm{ml}$ salmon sperm DNA. Hybridization buffer was replaced by fresh buffer containing $0.2 \mathrm{ng} / \mathrm{ml}$ DIG-labeled probe (either human Alu or mouse SINE B1/B2) before target DNA and probe were denatured for $5 \mathrm{~min}$. at $95^{\circ} \mathrm{C}$. Hybridization was carried out for $16 \mathrm{~h}$ at $42^{\circ} \mathrm{C}$ in a wet chamber. Slides were washed twice in SSC for $5 \mathrm{~min}$ at RT and twice for $10 \mathrm{~min}$. at $42^{\circ} \mathrm{C}$ in $0.1 \%$ SSC. Signals were detected using anti-DIG alkaline phosphatase conjugated Fab fragments (Roche, Germany) and nitroblue tetrazolium (NBT)/5-bromo-4chloro-3-indolyl phosphate (BCIP) (Roche, Germany) as a substrate.

\section{Biochemistry}

To analyze the extracellular matrix in the hydrogels the glycosaminoglycan (GAG) and collagen content was determined in the digested hydrogels and in the supernatants of the in vitro controls. Hydrogels were digested with $1 \mathrm{mg} /$ $\mathrm{ml}$ papain (Sigma-Aldrich) in 0.1 M Naacetate, 0.01 M Lcysteine, $0.05 \mathrm{M} \mathrm{Na}^{2}$-EDTA, and $0.2 \mathrm{M} \mathrm{NaCl}$ (pH 6.0) at $60^{\circ} \mathrm{C}$ overnight. Culture supernatants were used without digestion. The GAG content was measured by the restrictive version of the dimethylmethyleneblue (DMB) assay, including guanidinium hydrochloride in the protocol [27] and using chondroitin-4-sulfate (Sigma-Aldrich) as a standard. Proteoglycan content was expressed as $\mu \mathrm{g}$ GAG per complete hydrogel (with a hydrogel volume of approx. $0.25 \mathrm{ml}$ ). Total collagen content was measured by the hydroxyproline assay based upon alkaline hydrolysis and reaction with chloramine- $\mathrm{T}$ and dimethylbenzamidine using gelatine as a standard [28]; the resulting values were expressed as $\mu \mathrm{g}$ collagen per complete hydrogel. 


\section{Gene expression}

To recover the cells for gene expression analysis from monolayers, an aliquot of the initial cell suspension after detachment of the cells was used and the cell pellet lysed in RNeasy lysis-buffer (Qiagen; Hilden, Germany). In vitro cultured hydrogels were digested with proteinase $\mathrm{K}$ (3 mg/ml; Sigma-Aldrich) for about $10 \mathrm{~min}$. at $37^{\circ} \mathrm{C}$, the digest was centrifuged and the cell pellet lysed in RNeasy lysis-buffer [24]. The gel pieces retrieved from the mice were snap-frozen in liquid nitrogen, ground in a mortar, then dissolved in RLT-buffer for RNA extraction. Total RNA was extracted using the RNeasy mini kit plus DNase I digestion according to the manufacturer's instructions (Qiagen). Complementary DNA (cDNA) was obtained by reverse transcription of total RNA using the Reverse Transcriptase Core kit (Eurogentec; Cologne, Germany) with EuroScript reverse transcriptase (Moloney Murine leukemia virus reverse transcriptase, $50 \mathrm{U} / \mu \mathrm{l}$ ) and oligo-dT primers. Reverse transcription was performed in a total volume of $50 \mu \mathrm{l}$ at $48^{\circ} \mathrm{C}$ for $30 \mathrm{~min}$. in a thermocycler (Whatman Biometra; Goettingen, Germany).

Gene expression was analyzed by quantitative real-time PCR using an Applied Biosystems 7500 Fast Real-Time PCR System. The qPCR mastermix plus for SYBR green I (low ROX) kit from Eurogentec was used. Approximately $1 \mu \mathrm{g}$ of cDNA, $100-300 \mathrm{mM}$ primers and $2 \times$ reaction buffer were pipetted to a total volume of $25 \mu \mathrm{l}$. Primers defining exon-intron boundary spanning segments were newly designed with the primer express 2.0 software (Applied Biosciences; Darmstadt, Germany), except primers for type II collagen (COL2A1) [29], and were obtained from BIOTEZ. Sequences of all primers used are summarized in Table 1. Glyceraldehyde-3-phosphate dehydrogenase (GAPDH) was used as a reference gene. The PCR reaction was started with an initial enzyme activation step at $95^{\circ} \mathrm{C}$ for $10 \mathrm{~min}$ (hot start), followed by 40 cycles of $95^{\circ} \mathrm{C}$ for $15 \mathrm{sec}, 60^{\circ} \mathrm{C}$ for $30 \mathrm{sec}$, and $72^{\circ} \mathrm{C}$ for 30 sec. After the last cycle a dissociation curve was generated. Threshold cycles ( $\mathrm{Ct}$ values) were determined using Sequence Detection System software (Applied Biosystems). Ct value of the reference gene was subtracted from the $\mathrm{Ct}$ value of the gene of interest $(\mathrm{dCt})$ and the relative expression was expressed as $2^{\text {-dCt }}$. Median expression was calculated and is displayed in box plots relative to the median expression of the initial cell population. The boxes represent $25 \% / 75 \%$ percentiles, mean (dotted line) and median (solid line) values.

\section{Statistics}

Statistical analysis was performed using SigmaStat 3.0 (SPSS Science Software; Erkrath, Germany). Probability values p below 0.05 were considered to be significant.
Specific details of the applied statistical functions are given in the figures respectively the corresponding figure legends. In general, both median and mean are documented, considering the typically high variability of human cell cultures.

\section{Results}

The underlying philosophy of the experiments was to provide a continuous data set for the behavior of the intervertebral disc cells related to the hydrogel environment, as displayed during the cell expansion, after harvest into secondary culture as a monolayer or as an in vitro 3D (hydrogel) culture, and finally isolated in the ectopic environment (within the hydrogel) of the subcutaneous location in the mouse.

\section{Rheological measurements}

Rheological measurements were performed with nascent gel formulations produced out of the dual chamber syringe as described previously [24]. One measurement is exemplified in Figure 1. The gelation process of the gel is completed after approximately 2 - 3 minutes; thereafter the visco-elastic properties of the hydrogel remain nearly constant.

A total of 24 finished products were analyzed (Table 2). Thirteen measurements were performed with freshly prepared hydrogel components, five measurements were performed after storage gel components at $4^{\circ} \mathrm{C}$ for $24 \mathrm{~h}$ and six measurements were performed after storing the gel components at $4^{\circ} \mathrm{C}$ for $72 \mathrm{~h}$. The hydrogel production process could be successfully standardized; thus hydrogels with reproducible visco-elastic properties can be produced. There was no significant difference in storage modulus G' (measurements 1-3) and loss modulus G" (measurements 4-6) between the three time points (Table 2). Initial storage of the liquid gel precursors for $72 \mathrm{~h}$ does not alter the visco-elastic properties of the polymerized hydrogel. This storage time was chosen to simulate the necessary transportation of the hydrogel components to the user after preparation of the gel solution/cells mix.

\section{In vitro reference culture}

In an in vitro reference culture the alterations in gene expression of the matrix genes collagen type I, type II and aggrecan were analyzed at different time points during the culture of disc cells in monolayer and hydrogel cultures. This data set expression was compared to the expression data of the initial cell population (P0) used for the subculture (Figure 2). Collagen type I expression was almost unchanged over time and independent from culture type. Collagen type II expression decreased in monolayer cultures, at the end of the 14 days monolayer culture the 
Table 1 PCR primers

\begin{tabular}{|c|c|c|c|}
\hline Gene & Forward/reverse & Product Length & Accession \# \\
\hline \multirow[t]{2}{*}{$\mathrm{GAPDH}^{[a]}$} & AGAAAAACCTGCCAAATATGATGAC & 126 & NM_002046.3 \\
\hline & TGGGTGTCGCTGTTGAAGTC & & \\
\hline \multirow[t]{2}{*}{$\mathrm{COL} 1 \mathrm{~A} 2^{[a]}$} & GCTGGCAGCCAGTTTGAATATAAT & 78 & NM_000089.3 \\
\hline & CAGGCGCATGAAGGCAAGT & & \\
\hline \multirow[t]{2}{*}{$\mathrm{COL} 2 \mathrm{~A} 1^{[\mathrm{b}]}$} & AGAGGTATAATGATAAGGATGTGTGGAAG & 83 & NM_001844.3 \\
\hline & GTCGTCGCAGAGGACAGTCC & & \\
\hline \multirow[t]{2}{*}{$\mathrm{ACAN}^{[\mathrm{a}]}$} & TGCATTCCACGAAGCTAACCTT & 84 & NM_013227 \\
\hline & GACGCCTCGCCTTCTTGAA & & \\
\hline \multirow[t]{2}{*}{$\mathrm{HAS} 2^{[a]}$} & GACCCGTTGGAGCAAGTCCTA & 151 & NM_005328.2 \\
\hline & ACCCCGGTAGAAGAGCTGGAT & & \\
\hline \multirow[t]{2}{*}{$\mathrm{HAS}^{[\mathrm{a]}]}$} & CGGTGGCTCAACCAGCAA & 151 & AF234839 \\
\hline & GTATAACCGTGGCAATGAGGAAGA & & \\
\hline \multirow[t]{2}{*}{$\overline{\mathrm{SOX9}} \mathrm{g}^{[a]}$} & ATCCAAGCGCATTACCCACT & 132 & NM_000346.3 \\
\hline & CGATTCTCCATCATCCTCCAC & & \\
\hline
\end{tabular}

a Primers designed in-house using Primer Express 2.0 software

b [29]

median expression was 4-fold less (mean 18-fold) than the expression in the P0 cells. In contrast, in hydrogels the expression at all three time points was higher than in the P0 cells (median: 2 - 5-fold; mean: 1,7 - 11-fold). Although these differences were not statistically significant due to restricted number of patients $(n=4)$ and high individual variations a clear tendency was visible: the hydrogel culture improved the collagen type II expression compared to the monolayer (median: 10-fold, mean: 200-fold higher at day 14). Aggrecan expression was less effected by culture type. Nonetheless the same tendency for improved expression in hydrogels can be seen as for collagen type II mRNA expression.

\section{Mouse subcutaneous implants and in vitro cultured hydrogels}

The subcutaneous implantation into mice was intended to extend the in vitro data towards an in vivo application. In preliminary experiments, we found that gels without cells were completely resorbed within 8-12 weeks post implantation in SCID mice (data not shown). In order to achieve rapid accumulation of extracellular matrix, we decided to inoculate the hydrogels with a relatively high cell density. At the same time, this high density would give us pharmacological information on overdosing effects concerning the viability of cells in the gel under the presumed restrictive nutritional

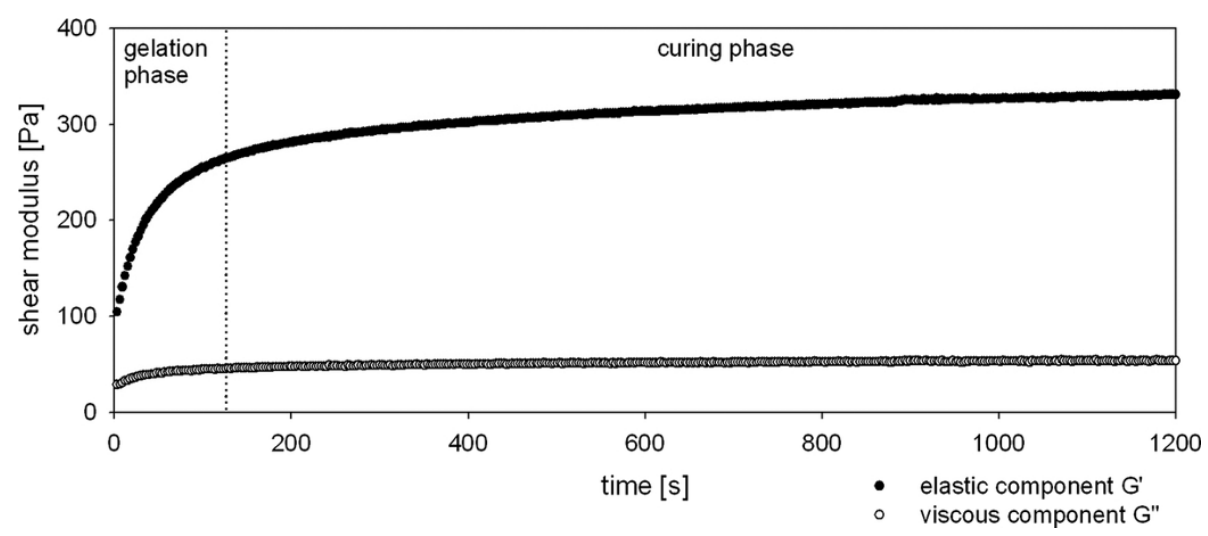

Figure 1 Gelation process of the hydrogel. The graph depicts the gelation process of the hydrogel separated into two phases, the gelation and the curing phase. The two gel components were mixed and injected onto the bottom plate of the rheometer using a dual-chamber applicator syringe. After adjusting the upper plate, the measurement started approximately $30 \mathrm{sec}$ after gel mixture. The experimental conditions of the gel formation were: temperature $37^{\circ} \mathrm{C}$, shear strain $0.05 \%$, shear stress $1 \mathrm{~Pa}$, oscillation frequency $1 \mathrm{~Hz}$. Storage modulus $\mathrm{G}^{\prime}$, representing the elastic component of the gel, and loss modulus $\mathrm{G}^{\prime \prime}$, representing the viscous component of the gel, were calculated every third second. The gelation is completed after approximately 2-3 minutes; thereafter the visco-elastic properties of the hydrogel are constant. 
Table 2 Effect of pre-gelation storage time at $4^{\circ} \mathrm{C}$ on the visco-elastic properties of the hydrogel

\begin{tabular}{cccc}
\hline storage time & $\mathbf{0 ~} \mathbf{h}$ & $\mathbf{2 4} \mathbf{~}$ & $\mathbf{7 2} \mathbf{~}$ \\
\hline measurement \# & 1 & 2 & 3 \\
\hline number of hydrogels & 13 & 5 & 6 \\
\hline mean G'[Pa] & 265.2 & 377.8 & 187.7 \\
\hline standard deviation & 158.1 & 78.1 & 156.4 \\
\hline measurement \# & 4 & 5 & 6 \\
\hline number of hydrogels & 13 & 5 & 6 \\
\hline mean G'[Pa] & 39.7 & 28.9 & 25.0 \\
\hline standard deviation & 22.6 & 10.3 & 9.8 \\
\hline
\end{tabular}

Storage modules $\mathrm{G}^{\prime}$ and loss modules $\mathrm{G}^{\prime \prime}$ were determined from hydrogels produced immediately after preparing the gel components $(0 \mathrm{~h})$, and after storing the liquid gel components for $24 \mathrm{~h}$ or $72 \mathrm{~h}$ at $4^{\circ} \mathrm{C}$. Mean value comparison using ANOVA showed no difference of storage modules between measurements \# 1 - 3 and no difference of loss modules between measurements \# 4 - $6(P<0.05)$

environment within the subcutis as compared to the unlimited supply in culture medium. The entire in vivo experiment was performed with cryo-conserved, in secondary culture expanded disc cells from six different patients, similar as planned to the future therapeutical use.

Upon injection, the gel polymerized instantly and produced approximately $0.25-\mathrm{ml}$ globules that remained stable and visible during the entire 14 days of incubation in the animal (Figure 3A). For comparison, cell-laden hydrogels were maintained in vitro in culture for the same time period.

At harvest, the implants presented as a separate entity within the animals. The opaque gels could be peeled out of the subcutis without any damage (Figure 3B). In contrast to their initial plasticity, the gels were solid to the grip and displayed some elasticity and structural stability.

Concerning the hydrogel itself, histological inspection revealed a rather homogenous distribution of the disc cells within the hydrogel (Figure 4A-C). There were no obvious regions with particular features such as higher densities of viable cells at the edge or the centre. To verify the presence and the distribution of human cells in the transplants a species specific genomic hybridization was performed. It was possible to discriminate between cells of human origin and cells of murine origin. Mouse cells are predominantly found at the edge of the xenotransplant (Figure 4E) (corresponding with the collagen type I staining); human cells are distributed evenly within the transplant (Figure 4D), confirming the above notion of the initial rather homogenous cell distribution. It appears that few cells with positive mouse Alu hybridization are also present within the hydrogel (Figure 4E). These might be false positive signals, as the probes to mouse Alu sequences tend to weakly bind to human Alu sequences. Since there are fewer mouse sequences than human Alu, the detection reaction with

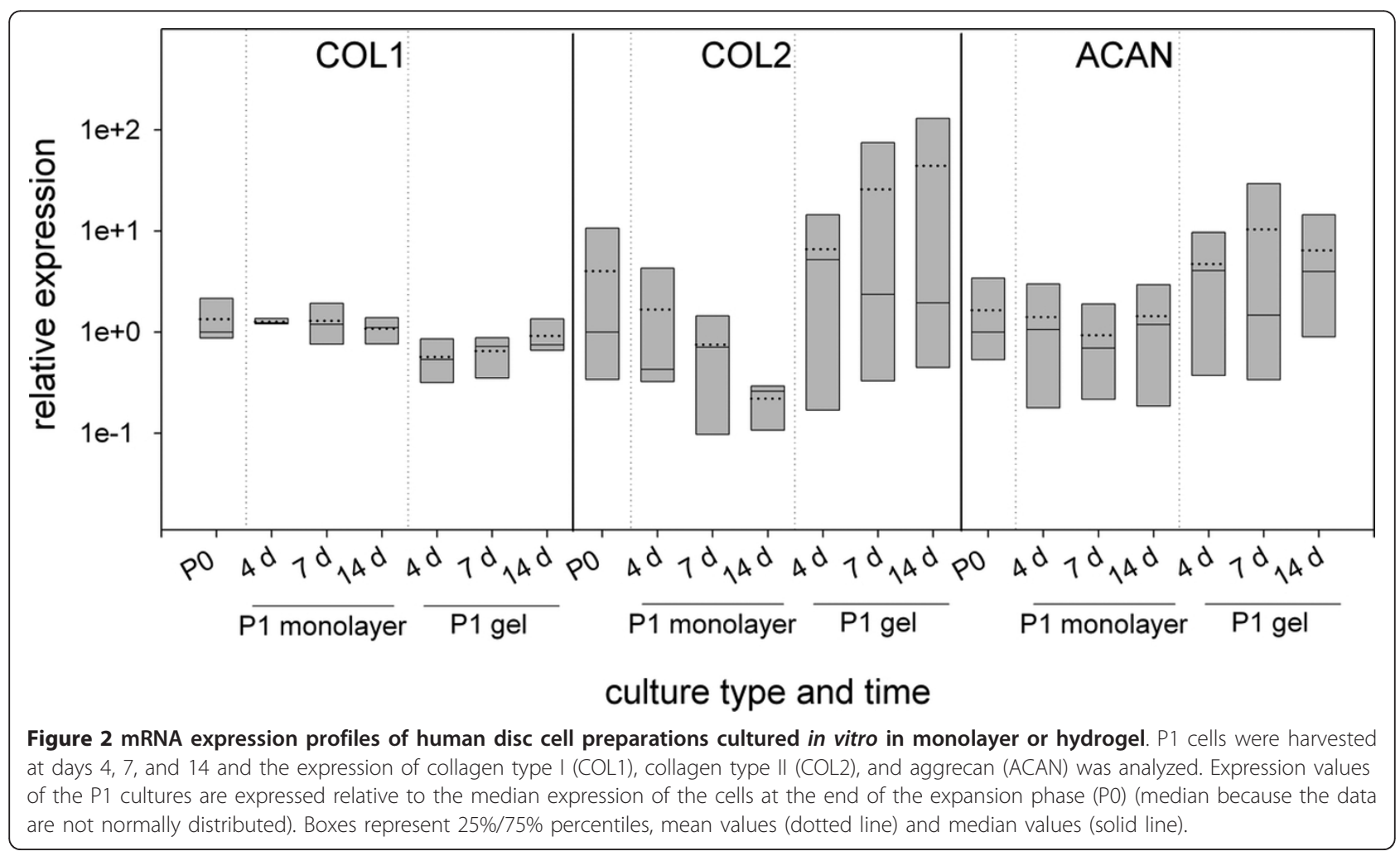



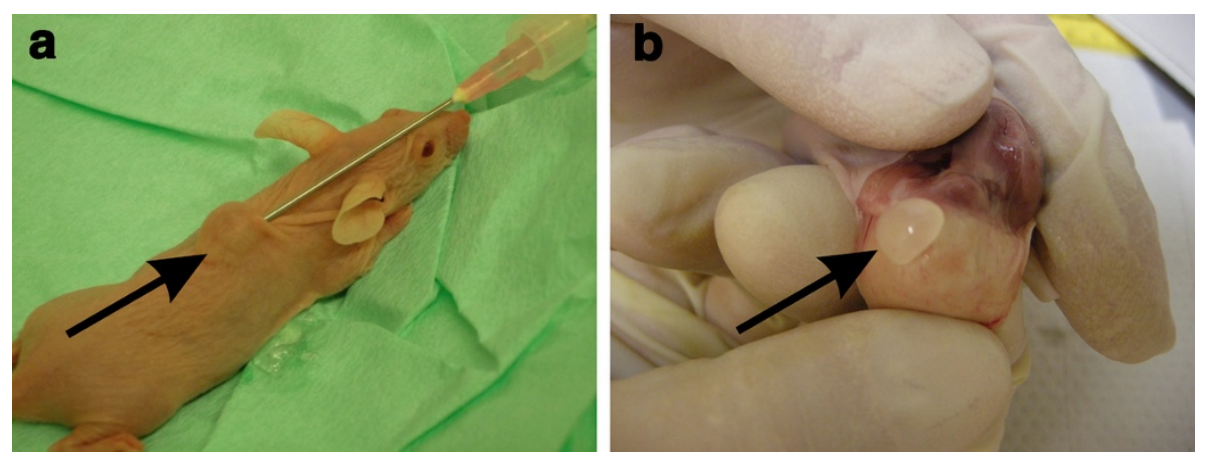

Figure 3 Injection and harvest of cell-loaded hydrogels. A: Injection from dual syringe and $1.3 \mathrm{~mm} \varnothing$ needle. The hydrogel (arrow) polymerizes upon injection and forms a stable subcutaneous protrusion. B: Hydrogel (arrow) at harvest. Note the absence of vascular and connective tissue ingrowth.

NBT/BCIP has to be intensified in order to visualize bound probes per nucleus in mouse cells. In this case, the unspecific binding on human cells may also appear and cause the present false positive signals on some cells.

The inner portions of the gel were positive for collagen type II and aggrecan immunostaining with the stain being either evenly distributed or clustered; collagen type I was found at the edges, and to a much lesser degree, in the centre of the gels. The accumulation of collagen type I at the edges of the in vivo transplants correlates with the appearance of a fibroblastic cell ring at the border of the gels, which could be seen in the in vitro cultured gels (data not shown).

In contrast to these staining patterns from the subcutaneous hydrogels, the in vitro cultured hydrogels displayed only very poor immunostaining for any antigen (data not shown, see also biochemical analysis in Figures 5 and 6). Upon macroscopic inspection, the hydrogels maintained in tissue culture for 14 days remained clear and soft with high plasticity, quite in contrast to the gels harvested from the mice. The results of the bulk biochemical
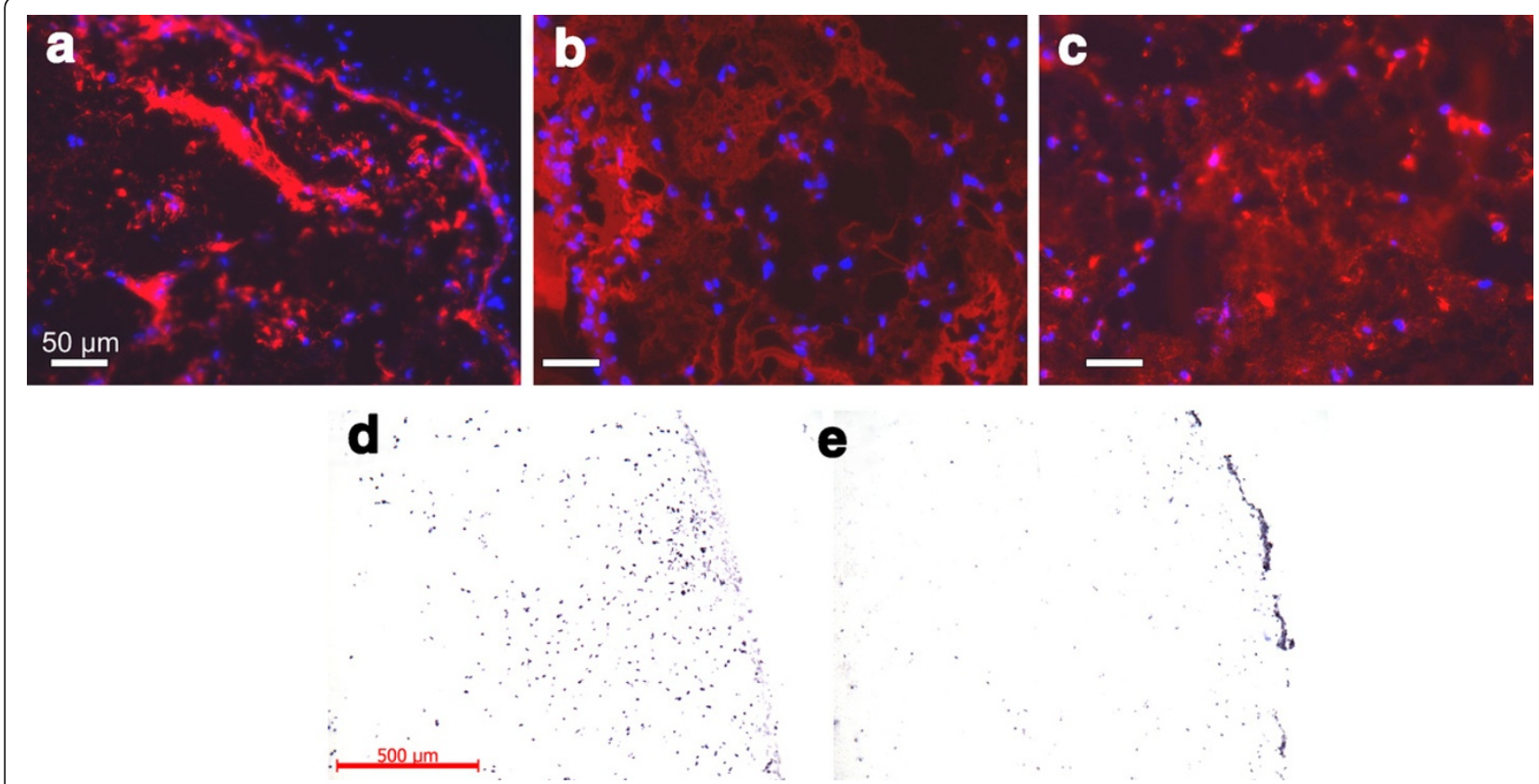

Figure 4 Immunohistochemistry staining and species specific genomic in situ hybridization of an implanted hydrogel upon harvest. A: Detection of collagen type I; B: collagen type II; C: aggrecan by immunohistochemistry. The blue dots are the DAPI stained nuclei. Note the even distribution of the nuclei across the samples, except for the edge in A. Species specific in situ hybridization discriminates between cells of human origin (D) and cells of murine origin (E). Mouse cells are predominantly found at the edge of the xenotransplant, human cells are distributed evenly within the transplant. 


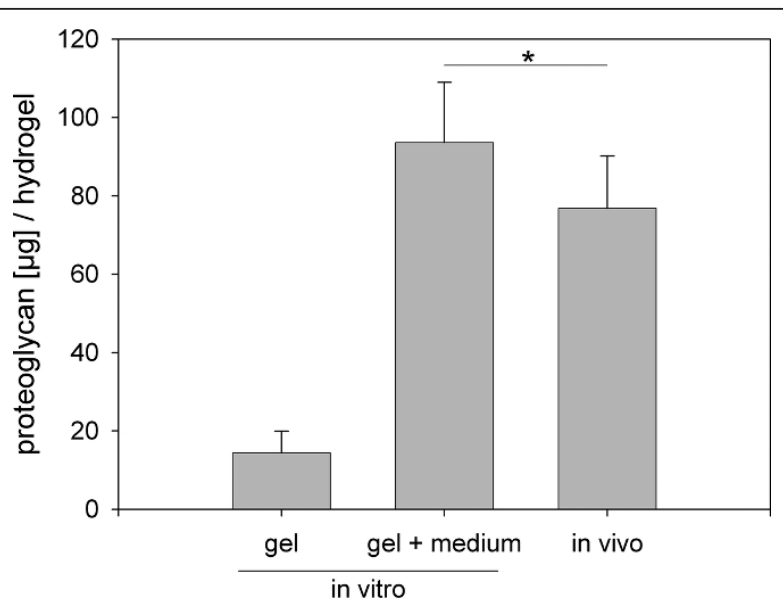

Figure 5 Glycosaminoglycan production from cells of in vitro maintained and of in vivo implanted hydrogels. Note that most of the glycosaminoglycans (GAG) from the in vitro cultures were found in the culture medium (middle bar). Mean values $(n=6)$ and standard deviation are shown. In vitro gel: GAG content of the in vitro cultured hydrogel; in vitro gel + medium: GAG content of the in vitro cultured hydrogel plus the GAG released into the combined medium supernatant from two weeks of culture; in vivo: GAG content of the in vivo implanted hydrogel. Statistic: paired $t$-test comparing the in vitro group (gel + medium) and the in vivo group, ${ }^{*}=p<0.05$

analysis may explain the contrasting findings. Cultured hydrogels contained more than five times less glycosaminoglycans (GAG) than implanted gels (Figure 5). The

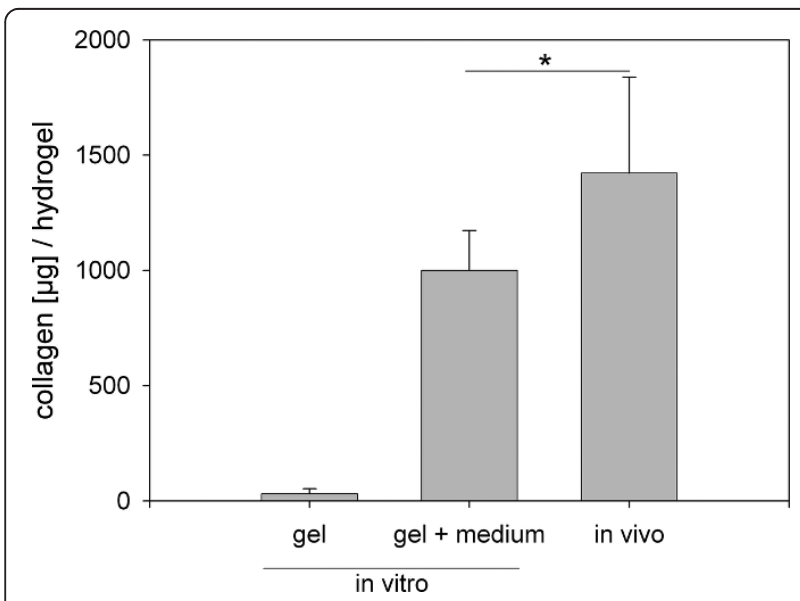

Figure 6 Cumulated collagen production from cells of in vitro maintained and of in vivo implanted hydrogels. Note that most of the collagens from the in vitro cultures were found in the culture medium (middle bar). Mean values $(n=6)$ and standard deviations are shown. In vitro gel: collagen content of the in vitro cultured hydrogel; in vitro gel + medium: collagen content of the in vitro cultured hydrogel plus the collagen released into the combined medium supernatant from two weeks of culture; in vivo: collagen content of the in vivo implanted hydrogel. Statistic: paired $t$-test comparing the in vitro group (gel + medium) and the in vivo group, $*^{*}=p<0.05$. collagen deposition in implanted gels was even 50-fold higher as in cultured gels (Figure 6). On the other hand, when the GAG and collagen content of the culture medium was taken into consideration, the total GAG and collagen output of the cultured cells was seemingly rather equal to that of the implanted cells (Figure 5 und6). In vitro cultured cells produced slightly but significantly more GAG than the subcutaneous cultured cells (1.2fold), conversely collagen accumulation was significantly higher in in vivo hydrogels (1.4-fold). Taken together, the entire process of matrix deposition in vivo could be considered to be more efficient towards formation of a functional tissue whereas in vitro conditions seemingly delayed that process.

At time of harvest, the deposition of ECM within the hydrogels was accompanied by a still active gene expression in the human cells, analyzed by RT-PCR for a number cartilage- and disc-specific factors including collagen types I, II, aggrecan, hyaluronan synthases- 2 and -3 (HAS2, HAS3), and SOX9 as a chondrocytic/disc cells key transcription factor (Figure 7). The median expression was compared to the expression levels of the initial cell population (P1) used for the production of the cell/hydrogel constructs (P2). As seen in the in vitro experiment, no significant changes in the transcription of genes coding for the matrix molecules collagen type I (COL1), type II (COL2), and aggrecan (ACAN) could be detected. Although both in vitro and in vivo hydrogels showed by trend higher expression levels for COL2 and ACAN, this effect was not statistically significant. Hydrogel culture had an extensive effect of the transcription of the hyaluronan-synthase genes and of the transcription factor SOX9. HAS2 and HAS3 expression was up-regulated to 4-5 orders of magnitudes in hydrogels cultures compared to the initial P1 cell population. Expression of both genes was slightly higher in in vitro hydrogels than in in vivo hydrogels (1.2-fold for HAS2 and 5-fold for HAS3), an effect being statistically significant for HAS2. Compared to P1 cells mRNA for SOX9 was found 80 times higher in in vitro hydrogels and about 160 times higher in in vivo transplants. As SOX9 expression accompanies chondrocytes and disc cells differentiation and cartilage/disc specific matrix production, the higher expression in hydrogels refers to a more differentiated phenotype compared the P1 cells.

\section{Discussion}

The present study generated data that support the idea of disc repair through the administration of intervertebral disc cells harvested from a disc prolaps and captured within an injectable hydrogel. In vitro and in vivo data point to a sustainable functionality of phenotypic disc cells within the hydrogel. Even though the present study did not investigate cell viability in particular, the gene 


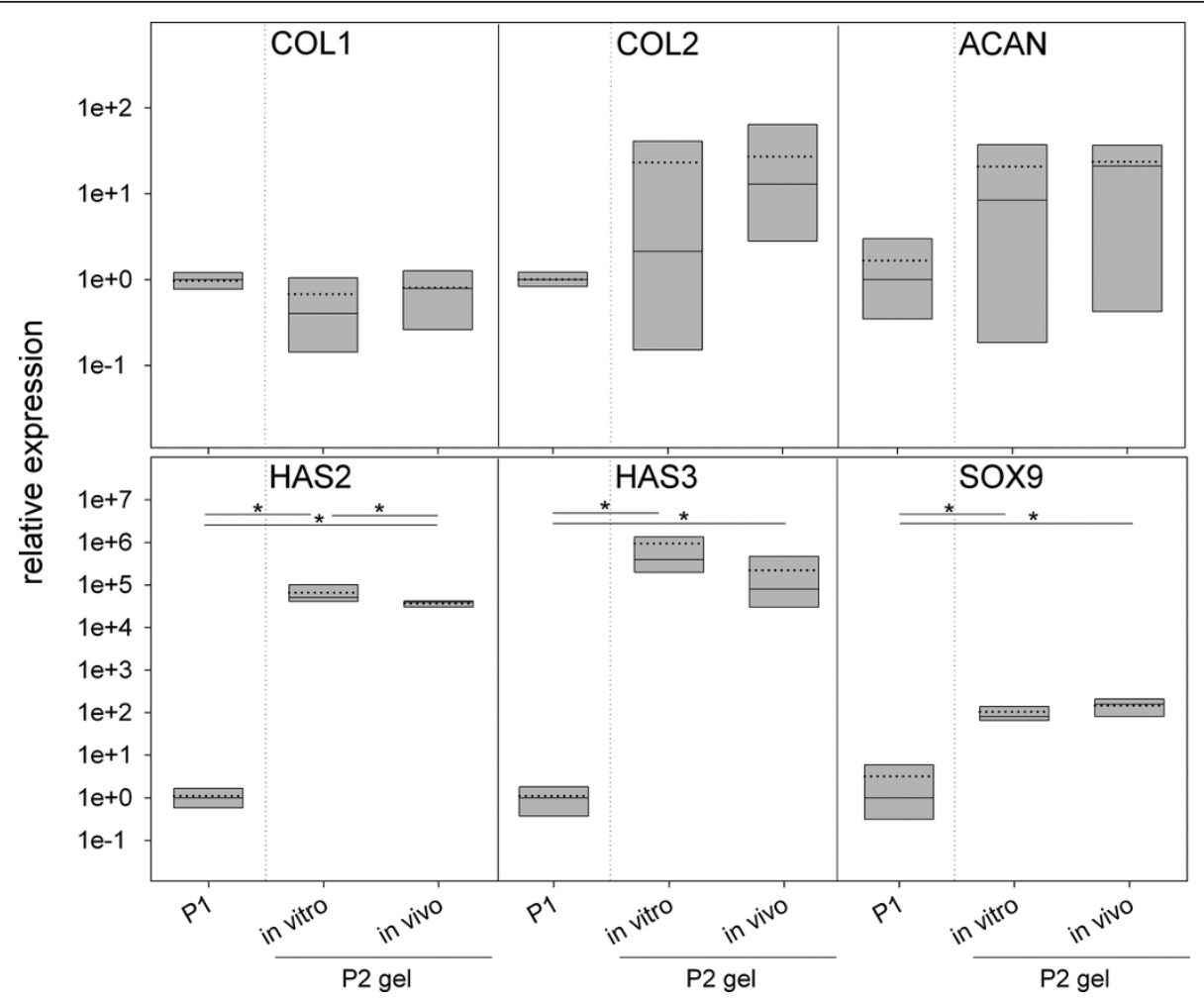

Figure 7 mRNA expression profiles of the human disc cells in vitro and in vivo. The expression of collagen type I (COL1), collagen type II (COL2), aggrecan (ACAN), hyaluronan-synthase-2 (HAS2), hyaluronan-synthase-3 (HAS3) and SRY (sex determining region Y)-box 9 (SOX9) was analyzed in P2 cells hydrogels cultured for two weeks in vitro or implanted in mice for two weeks and compared to the median expression of the P1 cells (median because not all data were normally distributed). Statistic: One Way repeated Measures Analysis of Variance was performed if the data of all groups were normally distributed, if not, Friedman Repeated Measures Analysis of Variance on Ranks was done. ${ }^{*}=p<0.05$.

Boxes represent 25\%/75\% percentiles, mean values (dotted line) and median values (solid line).

expression data indicate high viability in the present settings. Earlier work provided evidence that the viability of a number of cell types embedded into the hydrogel, including disc cells, is greater than 90\% [24]. In particular the rapid deposition of cartilagenous ECM and the significant levels of hyaluronan synthase isozymes- 2 and -3 , and SOX9 suggest cell functionalities towards those needed for the support of damaged intervertebral disc cores (the original nucleus pulposus). The reproducibility of the particular visco-elastic properties (rheological data) encourages administration of the gel mixture within the confinements of the damaged disc. The biomechanical properties of the hydrogel in intervertebral discs remain to be determined in specific studies to follow and have been in part presented by Beger [30].

Hydrogels are rapidly gaining attention as highly versatile carriers of bioactive compounds and cells in tissue engineering approaches [31-33]. We decided on a hydrogel formulation that is governed by aspects of drug safety and clinically established subcomponents. Contrary to many approaches, we refrained from choosing a base compound (such as collagen), that in itself may exert cell regulative properties towards chondrocyte-like cell types as they are found in intervertebral discs. This decision is based, among other considerations, on the well-known fact that chondrocytes and nucleus pulposus cells differentiate best in agarose, a compound that exerts no metabolic effect towards the cells and possesses a maximum of retentive properties towards newly synthesized ECM components, keeping the ECM in the vicinity of the cells [34,35]. Alginate is similar to agarose, in this aspect [36,37]. Serum albumin can be purchased as prescription drug. The chosen cross-linker, polyethylene glycol, has a long-standing history as a pharmaceutical additive and, as plasma expander, a proven history of non-toxicity. Albumin is readily degraded in vivo, with no toxic or otherwise critical intermediates or end products being generated in this process. Furthermore, in contrast to ester-based biopolymers, degradation of albumin does not set free acidic components. Thus a shift to lower $\mathrm{pH}$ is not expected during degradation of the hydrogel. The chemical polymerization reactions, starting from the maleimide activation of albumin and ending in the dual chamber application modality, possess no pharmaceutical problems, neither. 
The option to deliver, via an appropriate injection device, several components simultaneously, the therapeutic combination of cells, scaffold and regenerationpromoting substances (such as the hyaluronan applied here) was demonstrated in the presented animal experiment. No animal displayed any sign of distress, and recovery from anesthesia was speedy because of the short time needed for the injection. The injected mixture polymerized in situ and did not dissipate upon injection. There was a distinct border between the host tissue and the implant that could not be surmounted by invasive cell types of the animals' subcutis, just as published recently [5]. A subsequent histological examination of the in vivo maintained hydrogels revealed that the hydrogel prevented tissue ingrowth in part by its anti-angiogenic properties. This effect was then experimentally explored in various test systems and has been reported elsewhere [5].

One surprising key finding of these experiments was the presence of a balanced primary GAG and collagen biosynthesis by the cells for the cultured hydrogel as well as for the implanted hydrogels even though under the in vitro conditions, there was much less deposition of GAG and collagen within the gel structure. Regarding mRNA expression among the three groups of cells, P1cells, P2 in vitro hydrogel, and P2 in vivo hydrogel, there was a difference in favor of higher transcriptional activities in the hydrogels, but especially the difference between in in vitro and in vivo hydrogels resulted in only marginal differences from a functional standpoint in that both conditions led to approximately identical protein output by the cells. Baseline metabolic conditions may not be responsible for that effect since GAPDH expression levels were quite similar under all three conditions at time of harvest (data not shown). What differed, however, was the effective deposition of the produced ECM molecules within the hydrogels in vitro and in vivo.

Thus, the resulting difference in matrix deposition may have not been due to overall metabolic conditions but rather related to specific effects, such as differential matrix metalloprotease activities in vivo versus in vitro or the encapsulation by murine tissue. The higher deposition of GAG and collagen molecules in implanted gels may also arise from biomechanical stimulation of the construct in the in vivo situation. Additionally, the effect may be facilitated by the hypoxic conditions in a non-vascularised subcutaneous implant in mice $[38,39]$. The differential deposition of collagen type I and II within the in vivo incubated gels may represent another hint for specific processing of such matrix molecules or of spontaneous differential evolvement of cell types (chondrocytes/nucleus pulposus cells and fibrochondrocytes). These speculations need further investigations, though. The additional high levels of SOX9 message also differentiate cells from unspecific connective tissue cells and underscore their chondrogenic roots.

The pronounced expression of hyaluronan synthases supports the function of the hydrogel/cell mixture as disc regenerating element. HAS2 is the main producer of hyaluronan in chondrocytes, has been reported to be highly expressed in the intact nucleus pulposus $[40,41]$. HA is known to significantly contribute to osmotic pressure by locally binding large molar quantities of water [42]. The effect is enlarged by albumin, as in synovial fluid [43]. It has been investigated in many physiological contexts, including for example kidney, where HA supports water retentive activity [44], abdominal wall musculature, where HA supports the maintenance of the interstitial pressure [45], and in growth plates, where it facilitates in volume expansion of the hypertrophic cells $[46,47]$, to name some functions. HAS activity may therefore be chiefly responsible for the maintenance of the high hydrostatic pressure of a healthy disc.

\section{Conclusions}

The present study provides experimental evidence to support the idea of disc repair by intervertebral disc cells harvested from a disc prolaps and applied together with an injectable hydrogel. In vitro and in vivo data suggest that human disc cells even after a cryopreservation step are maintained in a functionally mature stage for at least two weeks when maintained in the hydrogel. Moreover, the molecular data derived from cells in hydrogel in vitro and in vivo corroborate the hypothesis that in vitro cultivation of cells in the hydrogel has some relevance for the development of such implants for clinical applications as well. In particular the rapid deposition of cartilagenous ECM and the significant levels of hyaluronan synthase isozymes- 2 and -3 of the propagated disc cells suggest functionalities needed for the support of damaged intervertebral discs. In continuation of these experiments, a large animal disc injury model for intervention in sheep based on hydrogel/cell composites application has already been completed. The data are currently evaluated, with early assessment indicating that upon further refinement the present approach will probably become suitable for disc repair in the near future.

\section{Acknowledgements}

This work was financially supported by the German Ministry of Education and Research (BMBF, grant no. 0313755).

\section{Author details}

${ }^{1} \mathrm{NMI}$ Natural and Medical Sciences Institute at the University of Tuebingen, Reutlingen, Germany. ${ }^{2}$ TETEC AG, Reutlingen, Germany. ${ }^{3} \mathrm{NMI} \Pi \mathrm{T} \mathrm{GmbH}$, Reutlingen, Germany. ${ }^{4} \mathrm{BG}$-Trauma Center, Tuebingen, Germany. ${ }^{5}$ Research Center for Experimental Orthopaedics, Orthopaedic University Hospital, 
Heidelberg, Germany. ${ }^{6}$ Centre for Medical Research (ZMF), Dept. Orthop. Surg., University Hospital, Tuebingen, Germany. ${ }^{7}$ Department of Biochemistry, Rush University Medical Center, Chicago, IL, USA.

\section{Authors' contributions}

KB conception and design of the study, analysis and interpretation of data, statistical analysis, drafting manuscript. CS carried out cell culture experiments, gene expression analysis, and histology. CO carried out biochemical assays. CG design and coordination of the study, revision of the manuscript. NL performed rheological measurements. AB selection of human donors, ethic approval for the use of human tissues. ES performed species specific in situ hybridization. WKA animal study, animal experiment approval, revision of the manuscript. JAM performed animal experiments, analysis and interpretation of data, drafting and revision of the manuscript. All authors read and approved the final manuscript.

\section{Competing interests}

$\mathrm{KB}$ and JAM have applied for a patent related to the use of the hydrogel material. CG holds shares in the TETEC AG.

Received: 3 January 2012 Accepted: 10 April 2012

Published: 10 April 2012

\section{References}

1. Meisel HJ, Ganey T, Hutton WC, Libera J, Minkus Y, Alasevic O: Clinical experience in cell-based therapeutics: intervention and outcome. Eur Spine J 2006, 15(Suppl 3):S397-S405.

2. Meisel HJ, Siodla V, Ganey T, Minkus Y, Hutton WC, Alasevic OJ: Clinical experience in cell-based therapeutics: disc chondrocyte transplantation A treatment for degenerated or damaged intervertebral disc. Biomol Eng 2007, 24(1):5-21.

3. Anderson PA, Rouleau JP: Intervertebral Disc Arthroplasty. Spine 2004, 29(23):2779-2786

4. Boyd L, Carter A: Injectable biomaterials and vertebral endplate treatment for repair and regeneration of the intervertebral disc. Eur Spine J 2006, 15(Suppl 3):S414-S421.

5. Scholz B, Kinzelmann C, Benz K, Mollenhauer J, Wurst H, Schlosshauer B: Suppression of adverse angiogenesis in an albumin-based hydrogel for articular cartilage and intervertebral disc regeneration. Eur Cell Mater 2010, 20:24-36, discussion 36-27.

6. Kobayashi S, Meir A, Kokubo Y, Uchida K, Takeno K, Miyazaki T, Yayama T, Kubota M, Nomura E, Mwaka E, et al: Ultrastructural analysis on lumbar disc herniation using surgical specimens: role of neovascularization and macrophages in hernias. Spine (Phila Pa 1976) 2009, 34(7):655-662.

7. Jia CQ, Zhao JG, Zhang SF, Qi F: Stromal cell-derived factor-1 and vascular endothelial growth factor may play an important role in the process of neovascularization of herniated intervertebral discs. J Int Med Res 2009, 37(1):136-144.

8. Ali R, Le Maitre CL, Richardson SM, Hoyland JA, Freemont AJ: Connective tissue growth factor expression in human intervertebral disc: implications for angiogenesis in intervertebral disc degeneration. Biotech Histochem 2008, 83(5):239-245.

9. Kokubo Y, Uchida K, Kobayashi S, Yayama T, Sato R, Nakajima H, Takamura T, Mwaka E, Orwotho N, Bangirana A, et al: Herniated and spondylotic intervertebral discs of the human cervical spine: histological and immunohistological findings in 500 en bloc surgical samples. Laboratory investigation. J Neurosurg Spine 2008, 9(3):285-295.

10. Volpi N, Schiller J, Stern R, Soltes L: Role, metabolism, chemical modifications and applications of hyaluronan. Curr Med Chem 2009, 16(14):1718-1745.

11. Heris HK, Rahmat M, Mongeau L: Characterization of a Hierarchical Network of Hyaluronic Acid/Gelatin Composite for use as a Smart Injectable Biomaterial. Macromol Biosci 2012, 12(2):202-210.

12. Kim IL, Mauck RL, Burdick JA: Hydrogel design for cartilage tissue engineering: a case study with hyaluronic acid. Biomaterials 2011, 32(34):8771-8782.

13. Nguyen LH, Kudva AK, Saxena NS, Roy K: Engineering articular cartilage with spatially-varying matrix composition and mechanical properties from a single stem cell population using a multi-layered hydrogel. Biomaterials 2011, 32(29):6946-6952.
14. Park K: Injectable hyaluronic acid hydrogel for bone augmentation. J Control Release 2011, 152(2):207.

15. Aulin C, Bergman K, Jensen-Waern M, Hedenqvist P, Hilborn J, Engstrand T: In situ cross-linkable hyaluronan hydrogel enhances chondrogenesis. J Tissue Eng Regen Med 2011, 5(8):e188-e196.

16. Park SH, Choi BH, Park SR, Min BH: Chondrogenesis of rabbit mesenchymal stem cells in fibrin/hyaluronan composite scaffold in vitro. Tissue Eng Part A 2011, 17(9-10):1277-1286.

17. Moss IL, Gordon L, Woodhouse KA, Whyne CM, Yee AJ: A novel thiolmodified hyaluronan and elastin-like polypetide composite material for tissue engineering of the nucleus pulposus of the intervertebral disc. Spine (Phila Pa 1976) 2011, 36(13):1022-1029.

18. Gloria A, Borzacchiello A, Causa F, Ambrosio L: Rheological characterization of hyaluronic acid derivatives as injectable materials toward nucleus pulposus regeneration. J Biomater Appl 2012, 26(6):745-759.

19. Calderon L, Collin E, Velasco-Bayon D, Murphy M, O'Halloran D, Pandit A: Type II collagen-hyaluronan hydrogel-a step towards a scaffold for intervertebral disc tissue engineering. Eur Cell Mater 2010, 20:134-148.

20. Toh WS, Lee EH, Guo XM, Chan JK, Yeow CH, Choo AB, Cao T: Cartilage repair using hyaluronan hydrogel-encapsulated human embryonic stem cell-derived chondrogenic cells. Biomaterials 2010, 31(27):6968-6980.

21. Gobbi A, Kon E, Berruto M, Filardo G, Delcogliano M, Boldrini L, Bathan L, Marcacci M: Patellofemoral full-thickness chondral defects treated with second-generation autologous chondrocyte implantation: results at 5 years' follow-up. Am J Sports Med 2009, 37(6):1083-1092.

22. Collin EC, Grad S, Zeugolis DI, Vinatier CS, Clouet JR, Guicheux JJ, Weiss P, Alini M, Pandit AS: An injectable vehicle for nucleus pulposus cell-based therapy. Biomaterials 2011, 32(11):2862-2870.

23. Elia R, Newhide DR, Pedevillano PD, Reiss GR, Firpo MA, Hsu EW, Kaplan DL, Prestwich GD, Peattie RA: Silk-hyaluronan-based composite hydrogels: A novel, securable vehicle for drug delivery. J Biomater Appl 2011.

24. Benz K, Freudigmann C, Mueller J, Wurst H, Albrecht D, Badke A, Gaissmaier C, Mollenhauer J: A polyethylene glycol-crosslinked serum albumin/hyaluronan hydrogel for the cultivation of chondrogenic cell types. Adv Eng Mater 2010, 12(9):B539-B551.

25. Linsenmayer TF, Hendrix MJ: Monoclonal antibodies to connective tissue macromolecules: type II collagen. Biochem Biophys Res Commun 1980, 92(2):440-446

26. Steck E, Burkhardt M, Ehrlich H, Richter W: Discrimination between cells of murine and human origin in xenotransplants by species specific genomic in situ hybridization. Xenotransplantation 2010, 17(2):153-159.

27. Chandrasekhar S, Esterman MA, Hoffman HA: Microdetermination of proteoglycans and glycosaminoglycans in the presence of guanidine hydrochloride. Anal Biochem 1987, 161(1):103-108.

28. Stegemann H, Stalder K: Determination of hydroxyproline. Clin Chim Acta $1967,18(2): 267-273$.

29. Gebhard PM, Gehrsitz A, Bau B, Soder S, Eger W, Aigner T: Quantification of expression levels of cellular differentiation markers does not support a general shift in the cellular phenotype of osteoarthritic chondrocytes. J Orthop Res 2003, 21(1):96-101.

30. Beger J, Wilke H-J, Fuchs H, Heuer F, Mollenhauer J, Benz K, Wurst H, Achatz G: A New Polymerizing Gel for Injection in the Nucleus Pulposus - Evaluation of the Biomechanical Behavior and the Extrusion Risk. International Society for the Advancement of Spine Surgery London, England ; 2009.

31. Schmidt JJ, Rowley J, Kong HJ: Hydrogels used for cell-based drug delivery. J Biomed Mater Res A 2008, 87(4):1113-1122.

32. Kim DN, Lee W, Koh WG: Micropatterning of proteins on the surface of three-dimensional poly(ethylene glycol) hydrogel microstructures. Anal Chim Acta 2008, 609(1):59-65.

33. Leach JB, Schmidt CE: Characterization of protein release from photocrosslinkable hyaluronic acid-polyethylene glycol hydrogel tissue engineering scaffolds. Biomaterials 2005, 26(2):125-135.

34. Benya PD, Shaffer JD: Dedifferentiated chondrocytes reexpress the differentiated collagen phenotype when cultured in agarose gels. Cell 1982, 30(1):215-224.

35. Buschmann MD, Gluzband YA, Grodzinsky AJ, Kimura JH, Hunziker EBT-LR: Chondrocytes in agarose culture synthesize a mechanically functional extracellular matrix. J Orthop Res 1992, 10(6):745-758. 
36. Hauselmann HJ, Fernandes RJ, Mok SS, Schmid TM, Block JA, Aydelotte MB, Kuettner KE, Thonar EJ: Phenotypic stability of bovine articular chondrocytes after long-term culture in alginate beads. J Cell Sci 1994, 107(Pt 1):17-27.

37. Bonaventure J, Kadhom N, Cohen-Solal L, Ng KH, Bourguignon J, Lasselin C, Freisinger P: Reexpression of cartilage-specific genes by dedifferentiated human articular chondrocytes cultured in alginate beads. Exp Cell Res 1994, 212(1):97-104.

38. Felka T, Schafer R, Schewe B, Benz K, Aicher WK: Hypoxia reduces the inhibitory effect of IL-1 beta on chondrogenic differentiation of FCS-free expanded MSC. Osteoarthr Cartil 2009, 17(10):1368-1376.

39. Coyle CH, Izzo NJ, Chu CR: Sustained hypoxia enhances chondrocyte matrix synthesis. J Orthop Res 2009, 27(6):793-799.

40. Nishida Y, Knudson CB, Nietfeld JJ, Margulis A, Knudson W: Antisense inhibition of hyaluronan synthase- 2 in human articular chondrocytes inhibits proteoglycan retention and matrix assembly. J Biol Chem 1999, 274(31):21893-21899.

41. Hiscock DR, Caterson B, Flannery CR: Expression of hyaluronan synthases in articular cartilage. Osteoarthr Cartil 2000, 8(2):120-126.

42. Peitzsch RM, Reed WF: High osmotic stress behavior of hyaluronate and heparin. Biopolymers 1992, 32(3):219-238.

43. Scott D, Coleman PJ, Mason RM, Levick JR: Interaction of intraarticular hyaluronan and albumin in the attenuation of fluid drainage from joints. Arthritis Rheum 2000, 43(5):1175-1182.

44. Hansell P, Goransson V, Odlind C, Gerdin B, Hallgren R: Hyaluronan content in the kidney in different states of body hydration. Kidney Int 2000, 58(5):2061-2068.

45. Zakaria ER, Lofthouse J, Flessner MF: In vivo effects of hydrostatic pressure on interstitium of abdominal wall muscle. Am J Physiol 1999, 276(2 Pt 2): H517-H529.

46. Gakunga PT, Kuboki Y, Opperman LA: Hyaluronan is essential for the expansion of the cranial base growth plates. I Craniofac Genet Dev Biol 2000, 20(2):53-63.

47. Pavasant $P$, Shizari $T$, Underhill CB: Hyaluronan contributes to the enlargement of hypertrophic lacunae in the growth plate. J Cell Sci 1996, 109(Pt 2):327-334.

\section{Pre-publication history}

The pre-publication history for this paper can be accessed here: http://www.biomedcentral.com/1471-2474/13/54/prepub

doi:10.1186/1471-2474-13-54

Cite this article as: Benz et al: Rheological and biological properties of a hydrogel support for cells intended for intervertebral disc repair. BMC Musculoskeletal Disorders 2012 13:54.

\section{Submit your next manuscript to BioMed Central and take full advantage of:}

- Convenient online submission

- Thorough peer review

- No space constraints or color figure charges

- Immediate publication on acceptance

- Inclusion in PubMed, CAS, Scopus and Google Scholar

- Research which is freely available for redistribution

Submit your manuscript at www.biomedcentral.com/submit
Biomed Central 